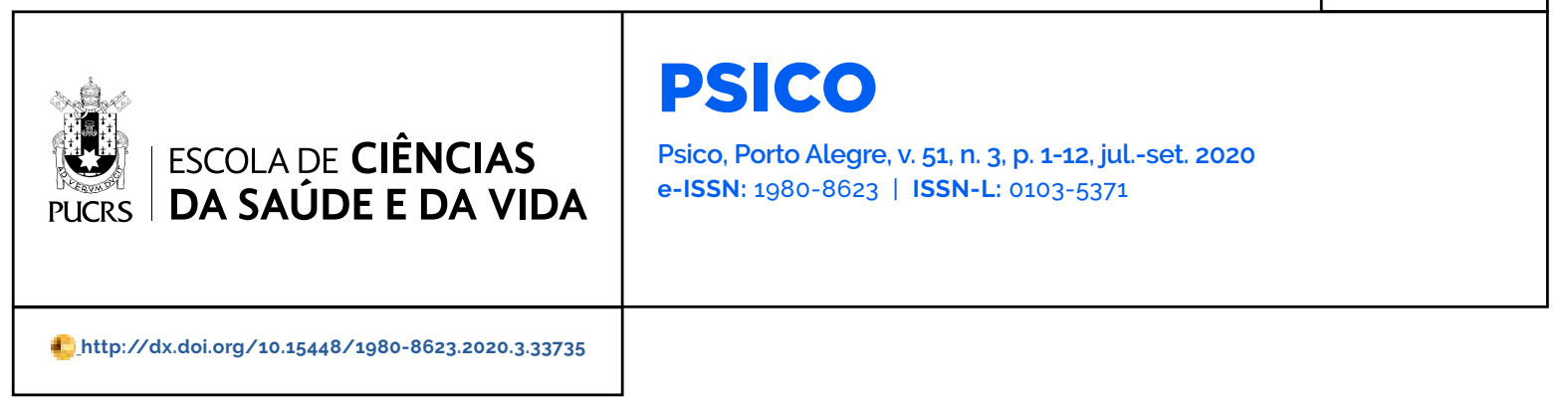

ARTIGOS

\title{
Construção e evidências de validade da Career Adapt-Abilities Scale (CAAS) brasileira para adolescentes
}

\author{
Construction and evidence of validity of Career Adapt-Abilities Scale (CAAS) \\ Brazilian form for adolescents \\ Construcción y evidencias de validez del Career Adapt-Abilities Scale (CAAS) \\ brasileña para adolescentes
}

\section{Larissa Sanford Ayres \\ Farina $^{1}$ \\ orcid.org/0000-0001-7313-2555}

lari.ayres.farina@gmail.com

\section{Roberta Pozzi \\ Kretzmann ${ }^{1}$}

orcid.org/0000-0001-9119-3714

robertakretzmann@gmail.com

\section{Letícia Garibaldi \\ Gasparetto $^{1}$}

orcid.org/0000-0002-5841-6928

leticiagasparetto@yahoo.com.br

\section{Gabriel dos Reis \\ Rodrigues $^{1}$}

orcid.org/0000-0002-0142-3123

reisrgabriel@gmail.com

Marúcia Patta Bardagi ${ }^{2}$

orcid.org/0000-0003-0224-2794

marucia.bardagi@gmail.com

\section{Cláudia Hofheinz}

Giacomoni ${ }^{1}$

orcid.org/0000-0002-9183-8591

giacomon@uol.com.br

Marco Antônio Teixeira ${ }^{1}$ orcid.org/0000-0001-7981-9788 mapteixeira.psi@gmail.com

Recebido em: 28/3/2019. Aprovado em: 30/4/2020. Publicado em: 21/01/2021

\section{(c) (1)}

Artigo está licenciado sob forma de uma licença Creative Commons Atribuição 4.0 Internaciona.
Resumo: A adaptabilidade de carreira é um tema amplamente estudado na área de carreira por sua relevância prática e teórica. O objetivo deste estudo foi testar uma versão em português brasileiro da Career Adapt-Abilities Scale (CAAS) com uma amostra de adolescentes, incluindo uma dimensão adicional chamada cooperação. Participaram 592 adolescentes (303 do sexo feminino) de escolas privadas $(n=275)$ e públicas $(n=317)$, em Porto Alegre, RS. Os adolescentes tinham entre 13 e 18 anos $(M=15.91$ e $D P=1,08)$. Em uma primeira subamostra aleatória ( $n=296)$, uma análise fatorial exploratória foi conduzida e resultou em uma solução de cinco fatores. Logo após, com o restante da amostra, foi realizada uma análise fatorial confirmatória, confirmando-se o modelo de cinco dimensões. Os resultados indicam que o instrumento testado possui uma estrutura dimensional compatível com o modelo teórico adotado e propriedades psicométricas aceitáveis, além de contar com o acréscimo da dimensão cooperação.

Palavras-chave: adaptabilidade, adolescentes, construção do teste

Abstract: Career adaptability is a theme widely studied in the area of career for its practical and theoretical relevance. The aim of this study was to test a Brazilian Portuguese version of the Career Adapt-Abilities Scale (CAAS) with a sample of adolescents, and including an additional dimension called cooperation. A total of 592 adolescents participated ( 303 female) from private $(n=275)$ and public schools $(n=317)$, in Porto Alegre. The adolescents were between 13 and 18 years old $(M=16,02$ e $S D=1,08)$. In the first random subsample $(n=296)$, an exploratory factor analysis was conducted and resulted in a five-factor solution. Afterwards, with the remainder of the sample, a confirmatory factor analysis was performed, ratifying the five-dimension model. The results indicate that the instrument tested has a dimensional structure compatible with the theoretical model adopted and acceptable psychometric properties, in addition to having the cooperation dimension added.

Keywords: adaptability, adolescents, test construction

Resumen: La adaptabilidad es uno de los temas ampliamente estudiados en el área de carrera, debido a su gran importancia práctica y teórica. El objetivo de este estudio es la creación de una versión brasilera de la escala CAAS, incluyendo la dimensión cooperación y destinada al público adolescente. Participaron 592 adolescentes (303 del sexo femenino) de escuelas privadas $(n=275)$ y públicas $(n=317)$, en Porto Alegre. Los adolescentes tenian entre 13 y 18 años ( $M=15.91$ y $D P=1,08)$. En una primera submuestra aleatoria $(n=296)$, se llevó a cabo un análisis factorial exploratorio que sugirió una solución de cinco factores. Luego, con el resto de la muestra, se realizó un análisis factorial confirmatorio, en el cual se confirmó el modelo de dimensiones. Por lo tanto, se puede llegar a una versión del CAAS con propiedades psicométricas compatibles con estudios anteriores para una muestra de adolescentes brasileños, con la adición de la dimensión cooperación. Palabras clave: adaptabilidad, adolescentes, construcción de test 
$\mathrm{Na}$ atualidade, uma nova maneira de se relacionar no contexto laboral surge frente à economia globalizada e à era digital. Inicia-se um novo modelo de trabalho em que os indivíduos passam a perceber a inconstância e as incertezas a respeito do futuro, as trajetórias deixam de ser estáveis e os trabalhadores passam a vivenciar várias mudanças ao longo de sua vida profissional. Essa nova realidade traz à tona a questão de que a carreira está ligada ao indivíduo e não ao seu local de trabalho (Barros, 2010; Duarte et al., 2010; Lassance \& Sarriera, 2012). A adaptabilidade de carreira refere-se aos recursos que um indivíduo possui para lidar com tarefas de desenvolvimento de carreira previsiveis e imprevisiveis, incluindo transições ocupacionais, sejam elas planejadas ou traumáticas, tal como a escolha por uma faculdade ou a perda de um emprego (Savickas, 1997. 2005, 2013). Suas dimensões estariam associadas às estratégias adaptativas que as pessoas se utilizariam para administrar tarefas relacionadas às suas carreiras (Savickas, 2013).

O conceito de adaptabilidade de carreira, elaborado por Super e Knasel (1981), surgiu em complemento ao de maturidade vocacional (ou maturidade de carreira), proposto anteriormente na teoria de Donald Super (Super \& Kidd, 1979). Para Super, a maturidade vocacional seria a prontidão do indivíduo para tomar decisões vocacionais, tipicamente durante a adolescência e a vida adulta jovem. O conceito de maturidade, no entanto, remete a uma ideia de desenvolvimento relacionado à idade cronológica e a uma decisão temporalmente circunscrita no ciclo vital, o que não é muito adequado para se descrever o desenvolvimento de carreira de adultos (Super \& Kidd, 1979). Em função disso, Super e Knasel sugeriram o termo adaptabilidade de carreira, que seria mais apropriado para descrever as atitudes de antecipação, planejamento, exploração e decisão necessárias para lidar com as cada vez mais frequentes transições (esperadas ou não) que ocorrem ao longo da trajetória ocupacional dos adultos.

Atualmente, o conceito de adaptabilidade de carreira tem sido aplicado também ao desenvolvimento de carreira de adolescentes e jovens adultos, pois entende-se que não há um estado definitivo de "prontidão" para as decisões ocupacionais, nem mesmo na adolescência. Para Savickas (1997, 2013), o desenvolvimento de carreira, da infância à idade adulta, exige do indivíduo a capacidade de responder de modo adaptativo a um contexto de baixa previsibilidade e que muda constantemente. As opções ocupacionais, para quem pode escolher, são cada vez maiores; ao mesmo tempo, não há garantias de sucesso ou mesmo de trabalho no futuro. Assim, para os adolescentes, a flexibilidade, ou adaptabilidade, seria uma capacidade necessária para que possam guiar suas escolhas em um cenário de incertezas.

Os recursos ou competências comportamentais que constituem conceitualmente a adaptabilidade de carreira compreendem quatro dimensões principais, a saber (Savickas \& Porfeli, 2012): preocupação (concern), controle (control), curiosidade (curiosity) e confiança (confidence), sendo referidos na literatura internacional como os quatro Cs da adaptabilidade de carreira. Essas quatro dimensões representam estratégias adaptativas que as pessoas utilizam para gerenciar tarefas relacionadas ao seu desenvolvimento de carreira (Savickas, 2013).

Pessoas com altas pontuações em preocupação demonstram capacidade para pensar sobre seu futuro vocacional, planejando e buscando estar preparadas para os próximos desafios. Pessoas com altas pontuações em controle têm autodisciplina, assumindo a responsabilidade por dar forma aos seus comportamentos e apresentando esforço e persistência. Pessoas com altas pontuações em curiosidade exploram as circunstâncias e buscam informações sobre oportunidades. Pessoas com pontuações altas em confiança têm certeza de que possuem a capacidade de resolver problemas e fazer o que precisa ser feito para superar obstáculos (Ambiel, 2014).

A adaptabilidade de carreira vem sendo investigada em diversos contextos. Esse construto tem se mostrado positivamente associado ao desempenho no trabalho e à capacidade de 
explorar e aproveitar recursos do trabalho (AffumOsei et al., 2019), a comportamentos proativos de carreira (Spurk, Volmer, Orth, \& Göritz, 2019), ao sucesso em carreira e a habilidades cognitivas (Rudolph, Lavigne, \& Zacher, 2017). Ainda, estudos têm demonstrado que a adaptabilidade está diretamente relacionada com proatividade, otimismo na carreira e orientação para cumprimento de objetivos, apresentandose inversamente correlacionada à insegurança profissional (Spurk, Kauffeld, Meinecke, \& Ebner, 2016; Tolentino et al., 2014).

A fim de nortear a mensuração da adaptabilidade de carreira, uma equipe internacional de psicólogos de 18 países trabalhou conjuntamente (Savickas \& Porfeli, 2012) para desenvolver a Career Adapt-Abilities Scale (CAAS), ou Escala de Adaptabilidade de Carreira. O primeiro passo na construção da CAAS foi especificar os principais aspectos e dimensões da adaptabilidade de carreira e definir um conjunto de 25 itens para cada dimensão. Com base em vários estudospiloto, o número de itens foi reduzido para 11 para cada uma das quatro dimensões. Esse conjunto de 44 itens representou o formulário de pesquisa da CAAS (ou CAAS 1.0). O formulário de pesquisa foi então administrado a diferentes populações de estudantes e trabalhadores em 13 dos 18 países que tinham participado anteriormente do estudo. Os dados foram analisados por Savickas e Porfeli (2012) com o objetivo de reduzir o número de itens para cada dimensão e examinar a estrutura fatorial proposta no modelo teórico de adaptabilidade.

Ao final, 24 itens foram selecionados para a versão CAAS Internacional (ou CAAS 2.0). A CAAS Internacional demonstrou consistência interna satisfatória para as quatro dimensões e para a pontuação total de adaptabilidade (Savickas \& Porfeli, 2012). Estudos posteriores realizados em outros países, alguns deles incluindo amostra adolescente, tais como Austrália (Tolentino et al., 2014), Filipinas (Tolentino, Garcia, Restubog, Bordia, \& Tang, 2013), Lituânia (Urbanaviciute, Kairys, Pociute, \& Liniauskaite, 2014), Ghana (Affum-Osei et al., 2019), China (Hou, Leung, Li, Li, \& Xu, 2012), Irã (McKenna, Zacher, Ardabili, \&
Mohebbi, 2016), Macau (Tien, Lin, Hsieh, \& Jin, 2014) obtiveram resultados similares aos de Savickas e Porfeli (2012) no que diz respeito às propriedades psicométricas do instrumento.

A versão brasileira da CASS internacional foi traduzida e validada para o Brasil por Teixeira, Bardagi, Lassance, Magalhães e Duarte (2012), utilizando uma amostra de estudantes universitários e trabalhadores. A estrutura fatorial foi bastante semelhante à obtida para os dados combinados dos 13 países relatada por Savickas e Porfeli (2012). Além disso, as estimativas de consistência interna para as subescalas e escala total variaram de boas a excelentes. Posteriormente, Audibert e Teixeira (2015) testaram uma versão brasileira da CAAS com o acréscimo de dois itens internacionais que não haviam sido incluidos na primeira versão da CAAS brasileira, além de introduzirem modificações na chave de respostas a fim de reduzir um efeito de teto verificado com a primeira versão. Os resultados confirmaram a estrutura fatorial do instrumento com os novos itens, mas a redução do efeito de teto não foi tão grande quanto o esperado.

Em 2012, McMahon, Watson, e Bimrose investigaram como um grupo de mulheres mais velhas lidou e se adaptou às mudanças e transições relacionadas à carreira, através do relato de histórias de transição e adaptabilidade. Esse estudo qualitativo foi conduzido com 36 mulheres australianas, inglesas e sul-africanas. Uma análise de dados dedutivos do subtema de adaptabilidade da carreira foi realizada, usando como descritores qualitativos as cinco dimensões de preocupação, controle, curiosidade, confiança e cooperação. Observou-se uma influência reciproca e clara entre as cinco dimensões, que se tornaram visiveis através da avaliação qualitativa. Esses cinco fatores não foram codificados igualmente e refletiram amplamente um processo multidimensional. Os resultados dessa pesquisa destacam uma dimensão a se considerar no atendimento em aconselhamento de carreira: a cooperação (McMahon et al., 2012).

Além das quatro dimensões da CAAS, a equipe internacional que desenvolveu o instrumento 
criou itens para essa outra dimensão chamada cooperação, embora ela não tenha sido incluida na versão final da CAAS, uma vez que o modelo de cinco fatores não apresentou resultados psicométricos adequados em todos os 13 países que fizeram parte da pesquisa (Nye, Leong. Prasad, Gardner, \& Tien, 2017). O fator cooperação tem como propósito avaliar aspectos interpessoais da adaptabilidade de carreira, como a capacidade de interagir com sucesso e trabalhar ao lado de outros. Esse fator facilitaria a adaptabilidade por propiciar maior adaptação a diversos contextos interpessoais.

Ainda sobre o fator cooperação, Pulakos, Arad, Donovan e Plamondon (2000) identificaram adaptabilidade interpessoal como uma das oito dimensões de adaptabilidade geral. As características dessa dimensão estão relacionadas a ser flexivel, ser receptivo ao lidar com as pessoas, conseguir ouvir e considerar diversos pontos de vista e opiniões, além de desenvolver relacionamentos efetivos e trabalhar harmonicamente em equipe. Ployhart e Bliese (2006) conceitualizam adaptabilidade interpessoal como um componente que faz parte de um traço de adaptabilidade global de ordem superior. Como os componentes de adaptabilidade interpessoal não estão representados dentro da operacionalização de quatro fatores da CAAS, parece pertinente que a adição do fator de cooperação seja necessária para representar de forma mais completa o construto de adaptabilidade de carreira.

Consistente com essa ideia, outros pesquisadores também têm incorporado aspectos interpessoais em suas avaliações de adaptabilidade de carreira (Rottinghaus, Buelow, Matyja, \& Schneider, 2011). Em 2015, os autores do instrumento original divulgaram uma versão da CAAS com os itens da dimensão cooperação, com o intuito de que novas pesquisas possam explorar os aspectos interpessoais da adaptabilidade de carreira e testar a medida proposta (Savickas \& Porfeli, 2015).

Nesse sentido, ao menos um estudo recente internacional procurou avaliar as propriedades psicométricas da CAAS acrescida da dimensão cooperação (Einarsdóttir, Vilhjálmsdóttir, Smáradóttir, \& Kjartansdóttir, 2015). Essa produção científica teve por objetivo validar a escala da CAAS, juntamente com a dimensão cooperação (CAAS-5), na Islândia. O estudo mostrou que tanto o modelo com quatro ou cinco fatores apresentou-se apropriado. Outros estudos também investigaram a cooperação como um elemento importante da adaptabilidade de carreira (McMahon et al., 2012; Nye et al., 2017). Tais estudos sugerem que o instrumento da CAAS-5 pode ser confiável e válido em culturas mais coletivistas, lugares em que o apoio entre os individuos é um fator importante na adaptação à carreira (Savickas \& Porfeli, 2015). Esses trabalhos evidenciaram que a CAAS é capaz de medir recursos pessoais acionados internamente, enquanto a CAAS-5 pode também ativar recursos externos dentro da comunidade.

Super e Knasel (1981) afirmam que o comportamento vocacional dos adolescentes difere do comportamento dos adultos já que os adolescentes, em sua grande maioria, têm uma experiência de trabalho mais incipiente e instável. Além disso, por estarem em uma importante fase de construção de identidade, adolescentes podem necessitar de auxilio para o desenvolvimento de competências associadas à adaptabilidade de carreira. Ainda, diversos estudos afirmam que o processo de adaptabilidade de carreira é particularmente importante no momento de uma escolha profissional ou da entrada no mundo do trabalho (Creed, Fallon, \& Hood, 2009; Gamboa, Paixão, \& Palma, 2014; Koen, Klehe, \& Van Vianen, 2013). Nestes casos, a avaliação rápida e precisa do construto de adaptabilidade de carreira mostrase fundamental ao se trabalhar com esse público.

Desse modo, o objetivo deste estudo é testar as propriedades psicométricas e obter evidências de validade da CAAS em uma amostra de adolescentes brasileiros, incluindo a dimensão cooperação. Portanto, essa versão da CAAS pode ser útil para pesquisas futuras que tenham por objetivo investigar o papel da adaptabilidade 
no desenvolvimento de carreira de jovens adolescentes.

\section{Método}

\section{Participantes}

Participaram 592 adolescentes (303 mulheres, 289 homens) de escolas privadas $(n=275)$ e públicas ( $n=317$ ) em Porto Alegre, Sul do Brasil. Os adolescentes tinham entre 13 e 18 anos, com média de idade de 15.91 anos $(D P=1,08)$, e cursavam o ensino médio. Desses jovens, 48 trabalhavam $(8,1 \%)$, sendo que 382 possuiam ensino técnico/médio completo (64.5\%).

\section{Instrumento}

Career Adapt-Abilities Scale and Cooperation

Scale (CAAS-5). Neste estudo foi utilizada a versão traduzida e adaptada para o Brasil da CAAS 1.0 (Savickas \& Porfeli, 2012), com os 44 itens originalmente concebidos pelo grupo de pesquisa internacional para medir as dimensões de preocupação, controle, curiosidade e confiança. Foram ainda acrescentados 11 itens da Cooperation Scale (Savickas \& Porfeli, 2015), que também foram traduzidos e adaptados pela equipe Brasil/ Portugal no período da construção da CAAS original. Os itens de cooperação faziam parte do conjunto de itens inicialmente criados pelo grupo internacional, embora tenham ficado de fora da versão internacional da CAAS, que contou com 24 itens ao final. Nesse conjunto de itens aplicados aos adolescentes já foram incluidos os dois itens faltantes na versão utilizada por Teixeira et al. (2012). Nota-se que foram aplicados os 55 itens originais da CAAS internacional, ao invés de apenas os itens testados por Audibert e Teixeira (2015). Dessa forma foi possivel ter mais opções de itens para compor a versão final do instrumento após as análises. Optou-se também por manter a chave de respostas original do instrumento.

O processo de tradução e de adaptação dos itens realizado no primeiro estudo com a CAAS no Brasil (Teixeira et al., 2012) é descrito sumariamente a seguir. Inicialmente, os itens originais em inglês foram traduzidos ao português brasileiro por um tradutor independente. O grupo de pesquisa responsável pela adaptação do instrumento no Brasil analisou, então, a adequação de cada item traduzido considerando a correspondência de conteúdo inglês-português e a sua pertinência enquanto indicador da dimensão de adaptabilidade à qual estava associado. Foram feitos ajustes na redação de alguns itens e, por consenso, foi escolhida a melhor versão em português para cada item. Posteriormente, a clareza dos itens e da chave de respostas foi testada em uma aplicação individual com cinco adolescentes, que não indicaram necessidade de modificações. Os indices de fidedignidade (alfa) das subescalas no estudo com a versão brasileira da CAAS aplicada a estudantes de ensino superior e trabalhadores foram: 0,77 (preocupação), 0,76 (controle), 0,81 (curiosidade), e 0,82 (confiança) (Teixeira et al., 2012).

\section{Procedimentos}

O presente estudo foi aprovado pelo Comitê de Ética do Instituto de Psicologia da Universidade Federal do Rio Grande do Sul e seguiu os procedimentos éticos recomendados (Parecer $\mathrm{n}^{\circ}$ 25000.089325/2006-58). Os instrumentos foram aplicados coletivamente em sala de aula, em horários disponibilizados pelas escolas que concordaram com a realização da pesquisa. Termos de consentimento foram enviados para casa a fim de que os pais ou responsáveis autorizassem a coleta de dados.

\section{Análise dos dados}

As análises foram realizadas em cinco etapas. Primeiro, estatísticas descritivas foram computadas para informações demográficas. Depois disso, a amostra $(N=610)$ foi dividida aleatoriamente em duas subamostras como procedimento recomendado para análise fatorial (Costello \& Osborne, 2005). Especificamente, uma análise fatorial exploratória (AFE) foi conduzida com a subamostra $A(n=296)$ e uma análise fatorial confirmatória (AFC) foi realizada com a subamostra B ( $n=296)$.

Na AFC foi utilizado o estimador WLSMV (Weighted Least Square Mean and Variance 
adjusted), adequado para variáveis ordinais como é o caso de itens com respostas em escala tipo Likert. Os indices de ajuste utilizados para avaliar a adequação do modelo incluiram a razão quiquadrado por graus de liberdade (2/gl), Índice de Ajuste Comparativo (CFI), Índice de Tucker-Lewis (TLI) e Aproximação de Erro Quadrado (RMSEA). Os valores tomados como indicadores de ajuste foram os seguintes: $2 / \mathrm{gl}=<3, \mathrm{CFl} / \mathrm{TLI}>=.95$ (ajuste excelente) ou .90 (bom ajuste); SRMR <.10 e RMSEA $<.08$. A carga fatorial mínima considerada foi de 0,30 (Hair, Black, Babin, Anderson, \& Tatham, 2010).

Finalmente, os coeficientes alfa das subescalas identificadas através da análise fatorial foram calculados com a subamostra A, para estimar a fidedignidade. A Análise Fatorial Exploratória e os coeficientes alfa foram calculados usando o software R. A Análise Fatorial Confirmatória foi conduzida por meio do Software Mplus Versão 8.0 (Muthén \& Muthén, 2017).

\section{Resultados}

\section{Análise Fatorial Exploratória}

A análise Principal Axis Factoring (Hair et al., 2010) foi empregada com o método de rotação Direct Oblimin. Análises sucessivas foram realizadas com o intuito de obter uma solução clara e coerente com as expectativas teóricas. Essas análises levaram à eliminação de itens com cargas ambíguas ou baixas (< 3), resultando em uma versão final de 25 itens, sendo cinco por dimensão.

As magnitudes das cargas fatoriais variaram entre .34 e .79. O coeficiente alfa para cada subescala variou entre .66 e .79. A medida Kaiser-Meyer-Olkin foi de 88 e o teste de Bartlett foi significativo, indicando a possibilidade de realização da análise fatorial. Cargas fatoriais, eigenvalues, porcentagem da variância comum explicada e coeficientes alfa são apresentados na Tabela 1

\section{Análise Fatorial Confirmatória}

Foi testado um modelo de segunda ordem, com um fator geral de adaptabilidade carregando em cada um dos cinco fatores de primeira ordem (preocupação, controle, curiosidade, confiança e cooperação), seguindo a ideia de um modelo hierárquico para a adaptabilidade de carreira identificado em estudo internacional prévio (Savickas \& Porfeli, 2012). Os resultados sugeriram um ajuste adequado do modelo, indicando que a estrutura fatorial poderia ser conceituada em um modelo de cinco fatores de primeira ordem contendo Subescalas de preocupação, cooperação, confiança, curiosidade e controle, e um fator de segunda ordem de adaptabilidade. Os indices de ajuste observados foram: $2 / \mathrm{gl}=1,73, p<0,001$, $\mathrm{CFI}=0.95, \quad \mathrm{TLI}=0.94, \mathrm{RMSEA}=0,05,90 \% \mathrm{Cl}$ RMSEA $=[0,042,0,057]$. A magnitude das cargas fatoriais variou entre .46. e .84. Cargas fatoriais e erros padrão são apresentados na Tabela 2.

\section{Discussão}

A partir dos resultados relatados, pôde-se chegar a uma versão da CAAS com propriedades psicométricas compativeis com estudos anteriores (Maggiori, Rossier, \& Savickas, 2017; Savickas \& Porfeli, 2012; Teixeira et al., 2012) para uma amostra de adolescentes brasileiros. Embora reduzida, a CAAS-5 manteve a estrutura de cinco fatores coerente com a base teórica de adaptabilidade. Ao se utilizar a segunda subamostra, as análises fatoriais confirmatórias demonstraram propriedade psicométricas adequadas para o modelo de cinco fatores hierárquico ajustado na versão proposta.

Apesar de apresentar propriedades psicométricas, em geral, de acordo com as validações anteriores da CAAS (Maggiori et al., 2017; Savickas \& Porfeli, 2012; Teixeira et al., 2012), a CAAS5 apresentou consistência interna um pouco abaixo do ideal (coeficiente alfa > 8), mas ainda satisfatória, para os escores dos cinco fatores e para o escore total da Adaptabilidade. Assim sendo, sugere-se que futuros estudos possam continuar testando a estabilidade da estrutura de cinco fatores, com enfoque na população adulta.

Com base nos dados levantados nesse trabalho, pode-se concluir que a CAAS-5, com acréscimo da dimensão cooperação, apresentou-se como um instrumento psicometricamente adequado para se medir o construto adaptabilidade para o 
TABELA 1 - Cargas Fatoriais para Análise Fatorial Exploratória dos 25 Itens da CAAS para Adolescentes

\begin{tabular}{|c|c|c|c|c|c|}
\hline \multirow{2}{*}{ Item } & \multicolumn{5}{|c|}{ Subescala } \\
\hline & $C R N$ & $\mathrm{COP}$ & $C F D$ & CUR & $C T L$ \\
\hline $\mathrm{CRN} 2$ & .75 & & & & \\
\hline $\mathrm{CRN}_{3}$ & .72 & & & & \\
\hline $\mathrm{CRN}_{4}$ & .67 & & & & \\
\hline CRN5 & .57 & & & & \\
\hline CRN6 & .55 & & & & \\
\hline $\mathrm{COP}_{4}$ & & .78 & & & \\
\hline $\mathrm{COP}_{3}$ & & .75 & & & \\
\hline $\mathrm{COP} 2$ & & .68 & & & \\
\hline COP5 & & .63 & & & \\
\hline COP9 & & .50 & & .34 & \\
\hline CFD10 & & & .75 & & \\
\hline CFD5 & & & .66 & & \\
\hline CFD2 & & & 64 & & \\
\hline CFD3 & & & .63 & & \\
\hline CFD4 & & .36 & .48 & & \\
\hline CUR1 & & & & .79 & \\
\hline CUR2 & & & & .55 & \\
\hline CURg & & & & .54 & \\
\hline CUR6 & .36 & & & .50 & \\
\hline CUR7 & & & & .47 & \\
\hline CTL8 & & & & & 64 \\
\hline CTL3 & & & & & 62 \\
\hline CTL7 & & & & & .60 \\
\hline CTL2 & & & & & .55 \\
\hline CTL9 & & & & & 34 \\
\hline Eigenvalue & 3.03 & 2.80 & 2.84 & 2.24 & 2.11 \\
\hline \% Variância Comum Explicada & 23 & 22 & 22 & 17 & 16 \\
\hline$\alpha$ & .72 & .79 & .76 & .70 & 66 \\
\hline
\end{tabular}

CRN = Subescala preocupação, $C O P=$ Subescala cooperação, CFD = Subescala confiança, CUR = Subescala curiosidade, $\mathrm{CTL}=$ Subescala controle. Casos abaixo de .30 foram omitidos. 
TABELA 2 - Cargas Fatoriais Padronizadas e Erros Padrão para os 25 Itens da CAAS para Adolescentes

\begin{tabular}{|c|c|c|c|}
\hline Dimensão & Item (indicadores de primeira ordem) & $\begin{array}{l}\text { Cargas } \\
\text { fatoriais }\end{array}$ & $E P$ \\
\hline \multirow[t]{5}{*}{ Preocupação } & CRN2. Perceber que meu futuro depende das escolhas de hoje & 69 & .043 \\
\hline & CRN3. Preparar-me para o futuro & .74 & .039 \\
\hline & $\begin{array}{l}\text { CRN4. Tomar consciência das escolhas educacionais e profissionais } \\
\text { que tenho de fazer }\end{array}$ & .76 & .038 \\
\hline & CRN5. Planejar como alcançar os meus objetivos & 69 & .045 \\
\hline & CRN6. Estar preocupado (a) com a minha carreira & .46 & .056 \\
\hline \multirow[t]{5}{*}{ Cooperação } & COP2. Agir de forma amigável & 63 & .041 \\
\hline & COP3. Dar-me bem com todo o tipo de pessoas & .72 & .039 \\
\hline & COP4. Cooperar com outros em projetos de grupo & .83 & .031 \\
\hline & COP5. Fazer a minha parte numa equipe & .84 & .041 \\
\hline & COP9. Compartilhar com os outros & .54 & .048 \\
\hline \multirow[t]{5}{*}{ Confiança } & CFD2. Ser responsável e fazer as coisas bem & .78 & .039 \\
\hline & CFD3. Desenvolver novas habilidades & 61 & .048 \\
\hline & CFD4. Dar sempre o meu melhor & .71 & .041 \\
\hline & CFD5. Superar obstáculos & .76 & .037 \\
\hline & CFD10. Ter confiança em mim mesmo (a) & .54 & .062 \\
\hline \multirow[t]{5}{*}{ Curiosidade } & CUR1. Explorar o ambiente à minha volta & .46 & .052 \\
\hline & CUR2. Procurar oportunidades para me desenvolver como pessoas & .80 & .040 \\
\hline & CUR6. Ser curioso (a) sobre novas oportunidades & 61 & .047 \\
\hline & CUR7. Imaginar possibilidades para o meu futuro & .59 & .053 \\
\hline & CURg. Analisar as minhas alternativas & .76 & .040 \\
\hline \multirow[t]{5}{*}{ Controle } & CTL2. Tomar decisões por mim mesmo (a) & .49 & .051 \\
\hline & CTL3. Assumir responsabilidade pelo que faço & .64 & .044 \\
\hline & CTL7. Pensar antes de agir & .61 & .046 \\
\hline & CTL8. Ser persistente e paciente & .51 & .053 \\
\hline & CTL9. Aprender como tomar decisões mais acertadas & .72 & .039 \\
\hline \multirow{6}{*}{$\begin{array}{l}\text { Dimensão } \\
\text { Adaptabilidade }\end{array}$} & Dimensão (indicadores de segunda ordem) & & \\
\hline & Preocupação & .75 & .044 \\
\hline & Cooperação & .65 & .049 \\
\hline & Confiança & .94 & .034 \\
\hline & Curiosidade & .78 & .040 \\
\hline & Controle & .86 & .039 \\
\hline
\end{tabular}


público adolescente. Espera-se que o instrumento possa estimular pesquisas que visem compreender como se desenvolve a adaptabilidade de carreira na adolescência e qual o papel da adaptabilidade nas decisões desse momento da vida, período em que se vivenciam muitas inquietações a respeito da carreira.

A nova escala pode, ainda, identificar rapidamente questões referentes à adaptabilidade e, assim, ajudar os orientadores de carreira a traçar intervenções assertivas. Em uma pesquisa recente, realizada por Cordeiro et al. (2017), a CAAS foi aplicada antes e depois das técnicas interventivas, auxiliando na evidência de eficácia das práticas utilizadas. Ademais, de acordo com Savickas (1990), uma técnica utilizada durante as intervenções consiste na utilização dos itens de um instrumento como pequenas intervenções durante as sessões de orientação. Assim sendo, os conselheiros discutiriam com seus orientandos o conteúdo dos itens da escala, o que poderia alavancar discussões sobre assuntos pertinentes em carreira e, assim, ajudar os clientes a desenvolverem competências e/ou atitudes que os impulsionem em direção à escolha profissional.

Segundo Savickas (2013), no processo de orientação profissional, primeiramente, objetiva-se possibilitar ao cliente manifestar sua narrativa de vida e refletir sobre o que deseja em sua carreira. Assim, o cliente observa, ressignifica episódios de sua história e repensa sobre seus planos futuros. Uma intervenção que leva em consideração a adaptabilidade de carreira e que se utiliza de instrumentos como a CAAS-5 pode auxiliar o cliente a clarificar e a contextualizar seus objetivos, na busca do desenvolvimento de maneiras para sua efetiva realização, mantendo sua motivação para alcançar a meta almejada (Savickas, 2013; Snyder, 2002).

Desse modo, com o auxílio do orientador, o indivíduo reflete sobre sua forma de pensar e agir, analisando como produz pensamentos voltados para o futuro (Savickas, 2013). Nessa etapa, busca-se ajudar o cliente a entender quais são suas principais motivações e interesses. Deste modo, uma aplicação da CAAS-5 nesse momento, a partir da leitura do conteúdo de seus itens, poderá impulsionar o cliente na direção de seu autoconhecimento em relação a sua carreira. Esse instrumento poderá ajudá-lo a refletir sobre suas preocupações em relação ao futuro, qual o controle que pode ter sobre ele, qual direção tomar na exploração de possibilidades que pode ter para colocar suas habilidades em prática e, por fim, o quanto confia em si mesmo para seguir adiante.

Em uma próxima fase, a intervenção é direcionada para auxiliar o cliente a investigar possiveis caminhos para colocar suas habilidades em prática. $\mathrm{O}$ indivíduo começa a ter insights e visualiza mais claramente os passos para atingir seus objetivos. Por meio de um processo de coconstrução e re-construção a pessoa reorganiza seus pensamentos, fica mais atenta ao seu processo e resignifica barreiras, movendo-se mais ativamente em direção ao que está buscando. Finalmente, ela pode rever, avaliar e ser autoeficaz o suficiente para se manter na busca de sua realização (Savickas et al., 2009: Snyder, 2002). Uma aplicação da CAAS-5 pós-intervenção pode auxiliar orientador e orientando a dar-se conta sobre o caminho que foi percorrido e sobre o quanto o cliente está ou não preparado para seguir adiante, dando foco e direcionamento na parte final das sessões.

No entanto, em concordância com Gleitman, Reisberg, e Gross (2009), é interessante notar que seria fundamental replicar o atual estudo, não somente com o intuito de se testar a constância das cinco dimensões, mas também com o objetivo de se ampliar a um maior número amostral (em diferentes estados do Brasil ou em diferentes paises, por exemplo). É fundamental que a dimensão cooperação seja avaliada em pessoas adultas no início de suas carreiras, em carreiras em momentos estáveis e, também, em transição. Dessa forma, conseguir-se-ia compreender a adaptabilidade de carreira de forma mais abrangente em relação às expectativas e comportamentos ativos das pessoas em suas profissões.

Além disso, recomenda-se novos trabalhos que analisem a validade externa desse instrumento com variáveis como autoestima, esperança, variáveis de personalidade, satisfação com a 
vida, autoeficácia em tomadas de decisão de carreira etc. (Hui, Yuen, \& Chen, 2018; Rudolph et al., 2017). Sendo assim, propõem-se a extensão dessa produção científica com o objetivo de se ampliar as evidências de validade da CAAS-5 para adolescentes e, assim, contribuir ainda mais para a pesquisa e a intervenção na área de carreira.

\section{Referências}

Affum-Osei, E., Antwi, C. O., Abdul-Nasiru, I., Asante E. A., Aboagye, M. O., \& Forkouh, S. K. (2019). Career adapt-abilities scale in Ghana: Psychometric properties and associations with individual-level ambidexterity and employees' service performance. Current Psychology. https://doi.org/10.1007/s12144019-00406-7

Ambiel, R. A. M. (2014). Adaptabilidade de carreira: uma abordagem histórica de conceitos, modelos e teorias. Revista Brasileira de Orientação Profissional, 15(1), 15-24. Recuperado de http://pepsic.bvsalud. org/scielo.php? script=sci_arttext\&pid=S1679$33902014000100004 \& \operatorname{lng}=$ pt\&tlng $=$ pt

Audibert, A. \& Teixeira, M. A. P. (2015). Escala de adaptabilidade de carreira: evidências de validade em universitários brasileiros. Revista Brasileira de Orientação Profissional, 16(1), 83-93. Recuperado de http://pepsic.bvsalud.org/pdf/rbop/v16n1/og.pdf

Barros, A. F. (2010). Desafios da Psicologia Vocacional: Modelos e intervenções na era da incerteza. Revista Brasileira de Orientação Profissional, 11(2), 165-175. Recuperado de http://pepsic.bvsalud.org/pdf/rbop/ v11n2/v11n2a02.pdf

Cordeiro, S. A., Taveira, M. C., Neves, L., Silva, A. D. Rodrigues, B., \& Lobo, C. C. (2017). Efeitos de uma Intervenção Psicológica Vocacional na Adaptabilidade de Carreira. Revista de Psicologia da Criança e do Adolescente, 8(1), 93-104. Recuperado de http://hdl. handle.net/1822/49173

Costello, A. B., \& Osborne, J. (2005). Best Practices in Exploratory Factor Analysis: Four Recommendations for Getting the Most from Your Analysis. Practical Assessment, Research \& Evaluation, 10(7), 1-9. Recuperado de https://pareonline.net/pdf/v1on7.pdf

Creed, P., Fallon, T., \& Hood, M. (2009). The relationship between career adaptability, person and situation variables, and career concerns in young adults. Journal of Vocational Behavior, 74(2), 219-229. https://doi.org/10.1016/j.jvb.2008.12.004

Duarte, M. E., Lassance, M. C., Savickas, M. L., Nota, L., Rossier, J., Dauwalder, J. P., Van Vianen, A. E. M. (2010). A Construção da Vida: Um Novo Paradigma para Entender a Carreira no Século XXI. Interamerican Journal of Psychology, 44(2), 392-406. Recuperado de https://Www.redalyc.org/pdf/284/28420641020.pdf
Einarsdóttir, S., Vilhjálmsdóttir, G., Smáradóttir, S. B., \& Kjartansdóttir, G. B. (2015). A culture-sensitive approach in the development of the Career AdaptAbilities Scale in Iceland: Theoretical and operational considerations. Journal of Vocational Behavior, 89, 172-181. https://doi.org/10.1016/i.jvb.2015.06.006

Gamboa, V., Paixão, O., \& Palma, A. (2014). Adaptabilidade de Carreira e Autoeficácia na Transição para o Trabalho: O papel da Empregabilidade Percebida Estudo com Estudantes do Ensino Superior. Revista Portuguesa de Pedagogia, 48(2), 133-156. https://doi. org/10.14195/1647-8614_48-2_7

Gleitman, H., Reisberg, D., \& Gross, J., (2009) Psicologia ( $7^{\mathrm{a}}$ ed.). Porto Alegre, RS: Artmed.

Hair, G., Black, B., Babin, B., Anderson, R., \& Tatham, R. (2010) Multivariate Data Analysis (7th ed.). Upper Saddle River, NJ: Pearson

Hou, Z.-J., Leung, S. A., Li, X., Li, X., \& Xu, H. (2012). Career Adapt-Abilities Scale_China Form: Construction and initial validation. Journal of Vocational Behavior, 80(3), 686-691. https://doi. org/10.1016/j.jvb.2012.01.006

Hui, T., Yuen, M., \& Chen, G. (2018). Career Adaptability, Self-Esteem, and Social Support Among Hong Kong University Students. The Career Development Quarterly, 66(2), 94-106. https://doi.org/10.1002/ cdq.12118

Koen, J., Klehe, U.-C., \& Van Vianen, A. E. M. (2013). Employability among the long-term unemployed: A futile quest or worth the effort? Journal of Vocational Behavior, 82(1), 37-48. https://doi.org/10.1016/j. jvb.2012.11.001

Lassance, M. C. P. \& Sarriera, J. C. (2012). Saliência do papel de trabalhador, valores de trabalho e desenvolvimento de carreira. Revista Brasileira de Orientação Profissional, 13(1), 49-61. Recuperado de http://pepsic.bvsalud.org/scielo.php? script=sci_ arttext\&pid=\$1679-33902012000100007

Maggiori, C., Rossier, J., \& Savickas, M. L. (2017). Career Adapt-Abilities Scale-Short Form (CAASSF): Construction and Validation. Journal of Career Assessment, 25(2), 312-325. https://doi. org/10.1177/1069072714565856

McKenna, B., Zacher, H., Sattari Ardabili, F., \& Mohebbi, H. (2016). Career Adapt-Abilities Scale-Iran Form: Psychometric properties and relationships with career satisfaction and entrepreneurial intentions. Journal of Vocational Behavior, 93, 81-91. https://doi. org/10.1016/j.jvb.2016.01.004

McMahon, M., Watson, M., \& Bimrose, J. (2012) Career adaptability: A qualitative understanding from the stories of older women. Journal of Vocational Behavior, 80(3), 762-768. https://doi.org/10.1016/j. jvb.2012.01.016

Muthén, L. K. \& Muthén, B. O. (2017). Mplus Statistical Analysis with Latent Variables User's Guide (Version 8). Los Angeles, CA: Muthén \& Muthén. 
Nye, C. D., Leong, F., Prasad, J., Gardner, D., \& Tien, H.-L. S. (2017). Examining the Structure of the Career Adapt-Abilities Scale: The Cooperation Dimension and a Five-Factor Model. Journal of Career Assessment, 26(3), 549-562. https://doi. org/10.1177/1069072717722767

Ployhart, R. E. \& Bliese, P. D. (2006). Individual adaptability (I-Adapt) theory: Conceptualizing the antecedents, consequences and measurement of individual differences in adaptability. In C. S. Burke, L. G. Pierce, \& E. Salas (Eds.), Understanding adapatility: A prerequisite for effective performance within complex environments (pp. 3-39). Amsterdam, the Netherlands: Elsevier

Pulakos, E. D., Arad, S., Donovan, M. A., \& Plamondon, K. E. (2000). Adaptability in the workplace:

Development of a taxonomy of adaptive performance. Journal of Applied Psychology, 85(4), 612. https://doi. org/10.1037/0021-9010.85.4.612

Rottinghaus, P. J., Buelow, K. L., Matyja, A., \& Schneider, M. R. (2011). The Career Futures InventoryRevised: Measuring dimensions of career adaptability Journal of Career Assessment, 20(2), 123-139. https:// doi.org/10.1177/1069072711420849

Rudolph, C. W., Lavigne, K. N., \& Zacher, H. (2017). Career adaptability: A meta-analysis of relationships with measures of adaptivity, adapting responses, and adaptation results. Journal of Vocational Behavior, 98 , 17-34. https://doi.org/1010.1016/j.jvb.2016.09.002

Savickas, M. L. (1990). The Career Decision-Making Course: Description and Field Test. The Career Development Quartely, 38(3), 275-284. https://doi. org/10.1002/i.2161-0045.1990.tb00388.x

Savickas, M. L. (1997). Career Adaptability: An Integrative Construct for LifeSpan, LifeSpace Theory The Career Development Quarterly, 45(3), 247-259. https://doi.org/10.1002/j.2161-0045.1997.tb00469.x

Savickas, M. L. (2005). The Theory and Practice of Career Construction. In S. D. Brown, \& R. W. Lent (Eds.), Career development and counseling: Putting theory and research to work (pp. 42-70). Hoboken, NJ: Wiley \& Sons.

Savickas, M. L. (2013). Career construction theory and practice. In S. D. Brown \& R. W. Lent (Eds.), Career Development and Counseling: Putting theory and research to work (2nd ed., pp. 147-183). Hoboken, NJ: Wiley \& Sons.

Savickas, M. L., Nota, L., Rossier, J., Dauwalder, J.-P., Duarte, M. E., Guichard, J., ... van Vianen, A. E. M. (2009). Life designing: A paradigm for career construction in the 21st century. Journal of Vocational Behavior, 75(3). 239-250. https://doi.org/10.1016/j.jvb.2009.04.004

Savickas, M. L. \& Porfeli, E. J. (2012). Career AdaptAbilities Scale: Construction, reliability, and measurement equivalence across 13 countries. Journal of Vocational Behavior, 80(3), 661-673. https:// doi.org/10.1016/j.jvb.2012.01.011

Savickas, M. L. \& Porfeli, E. J. (2015). The Career AdaptAbilities Scale + Cooperation Scale. Recuperado de http://www.vocopher.com/ms/cmic/CAAS+C.pdf
Snyder, C. R. (2002). Hope theory: Rainbows in the mind. Psychological Inquiry, 13(4), 249-275. https://doi. org/10.1207/S15327965PLl1304_01

Spurk, D., Kauffeld, S., Meinecke, A. L., \& Ebner, K. (2016). Why do adaptable people feel less insecure? Indirect effects of career adaptability on job and career insecurity via two types of perceived marketability. Journal of Career Assessment, 24(2), 289-306. https://doi.org/10.1177/1069072715580415

Spurk, D., Volmer, J., Orth, M., \& Göritz, A. S. (2019) How do career adaptability and proactive career behaviours interrelate over time? An inter and intraindividual investigation. Journal of Occupational and Organizational Psychology, 93(1), 158-186. https:// doi.org/10.1111/joop.12288

Super D. E. \& Kidd, J. M. (1979). Vocational maturity in adulthood: Toward turning a model into a measure. Journal of Vocational Behavior, 14(3), 255270. https://doi.org/10.1016/0001-8791(79)90054-X

Super, D. E. \& Knasel, E. G. (1981). Career development in adulthood: Some theoretical problems and a possible solution. British Journal of Guidance \& Counselling, 9(2), 194-201. https://doi. org/10.1080/0306.9888100760211

Teixeira, M. A. P., Bardagi, M. P., Lassance, M. C. P., Magalhães, M. de O., \& Duarte, M. E. (2012). Career Adapt-Abilities Scale-Brazilian Form: Psychometric properties and relationships to personality. Journal of Vocational Behavior, 80(3), 680-685. https://doi. org/10.1016/j.jvb.2012.01.007

Tien, H.-L. S., Lin, S.-H., Hsieh, P.-J., \& Jin, S.-R. (2014). The Career Adapt-Abilities Scale in Macau: Psychometric characteristics and construct validity. Journal of Vocational Behavior, 84(3), 259-265. https:// doi.org/10.1016/i.jvb.2014.01.005

Tolentino, L. R., Garcia, P. R. J. M., Lu, V. N., Restubog, S. L. D., Bordia, P., \& Plewa, C. (2014). Career adaptation: The relation of adaptability to goal orientation, proactive personality, and career optimism. Journal of Vocational Behavior, 84(1), 39-48. https://doi.org/10.1016/j.jvb.2013.11.004

Tolentino, L. R., Garcia, P. R. J. M., Restubog, S. L. D., Bordia, P., \& Tang, R. L. (2013). Validation of the Career Adapt-Abilities Scale and an examination of a model of career adaptation in the Philippine context. Journal of Vocational Behavior, 83(3), 410-418. https://doi. org/10.1016/j.jvb.2013.06.013

Urbanaviciute, I., Kairys, A., Pociute, B., \& Liniauskaite, A. (2014). Career adaptability in Lithuania: A test of psychometric properties and a theoretical model. Journal of Vocational Behavior, 85(3), 433-442. https:// doi.org/10.1016/j.jvb.2014 


\section{Endereço para correspondência:}

Larissa Sanford Ayres Farina

Universidade Federal do Rio Grande do Sul

Rua Ramiro Barcelos, 2600, sala 117

90035-003 - Porto Alegre, RS, Brasil

\section{Larissa Sanford Ayres Farina}

Psicóloga formada pela Universidade Federal do Rio Grande do Sul (UFRGS), em Porto Alegre, RS, Brasil; especialista em Gestão de Pessoas e em Dinâmica dos Grupos, Mestra e atual doutoranda do Programa de PósGraduação em Psicologia, também pela Universidade Federal do Rio Grande do Sul (UFRGS), em Porto Alegre. RS, Brasil.

\section{Roberta Pozzi Kretzmann}

Psicóloga. Especialista em Psicodiagnóstico e Avaliação Psicológica pelo Contemporâneo: Instituto de Psicanálise e Transdisciplinaridade (CIPT/RS). Mestre em Psicologia pela Universidade Federal do Rio Grande do Sul (UFRGS), em Porto Alegre, RS, Brasil. Membro do BioSig (Laboratório de Biossinais Cognitivos) da UFRGS.

\section{Letícia Garibaldi Gasparetto}

Graduada em Psicologia pela Universidade Federal do Rio Grande do Sul (UFRGS), em Porto Alegre, RS, Brasil; Especialista em Terapia Cognitivo-Comportamental pelo InTCC; Mestre em Desenvolvimento Humano pela UFRGS; doutoranda em Psicologia pela UFSC.

\section{Gabriel dos Reis Rodrigues}

Graduado em Psicologia pela Universidade Federal do Rio Grande do Sul (UFRGS), em Porto Alegre, RS, Brasil; mestrando em Psicologia pelo Programa de Pós-Graduação em Psicologia pela UFRGS, no Núcleo de Estudos e Intervenção em Carreira.

\section{Marúcia Patta Bardagi}

Professora adjunta do curso de Psicologia da UFSC. Psicóloga pela Universidade Federal do Rio Grande do Sul (UFRGS); Mestra e Doutora em Psicologia pelo Programa de Pós-Graduação em Psicologia também pela UFRGS, em Porto Alegre, RS, Brasil.

\section{Cláudia Hofheinz Giacomoni}

Professora do Departamento de Psicologia do Desenvolvimento e da Personalidade e do Programa de Pós-Graduação em Psicologia da Universidade Federal do Rio Grande do Sul (UFRGS), em Porto Alegre, RS, Brasil. Graduada em Psicologia pela PUCRS e Mestra e Doutora pela UFRGS

\section{Marco Antônio Teixeira}

Psicólogo formado pela UFRGS, Mestre e Doutor pelo Programa de Pós-graduação em Psicologia da Universidade Federal do Rio Grande do Sul (UFRGS), em Porto Alegre, RS, Brasil. Coordenador dos programas de extensão continuados Núcleo de Apoio ao Estudante da UFRGS (desde 2006) e Serviço de Orientação Profissional (desde 2012). 